\title{
Evaluación de la calidad de agua para riego y aprovechamiento del recurso hídrico de la quebrada Togllahuayco
}

\section{Water quality assessment for irrigation and use of the water resource of the Togllahuayco gorge}

\author{
Joffre Quinteros-Carabalí1 ${ }^{1}$, Javier Gómez-García ${ }^{1}$, Michelle Solano $^{1}$, Gabriela Llumiquinga ${ }^{1}$, Christian Burgos ${ }^{1}$, \\ David Carrera-Villacrés ${ }^{1,2,3}$
}

Autor para correspondencia: jaquinteros@uce.edu.ec

\footnotetext{
${ }^{1}$ Universidad Central del Ecuador. Carrera Ingeniería Ambiental, Facultad de Ingeniería en Geología, Minas, Petróleos y Ambiental, Ciudadela Universitaria, Quito 170129

${ }^{2}$ Universidad de las Fuerzas Armadas ESPE. Departamento de Ciencia de la Tierra y la Construcción. Av. Gral. Rumiñahui, Sangolquí, Ecuador
} ${ }^{3}$ Grupo de Investigación en Contaminación Ambiental (GICA)

\begin{abstract}
Resumen
El agua utilizada para riego con fines agrícolas tiene efectos importantes sobre la producción de cultivos, así como también sobre el deterioro químico del suelo. En este estudio se evalúa la cantidad y calidad de agua disponible para el riego de un cultivo sustentable de quinua en la quebrada Togllahuayco, parroquia Guangopolo, Ecuador. Esta quebrada dispone de un volumen de $23.347,95 \mathrm{~m}^{3}$ anuales de agua y solo requiere de $6.747,5 \mathrm{~m}^{3}$ anuales para el cultivo de quinua. Se tomaron muestras de agua y se midió la concentración de sodio, potasio, calcio, magnesio, carbonatos, bicarbonatos, sulfatos y cloruros y la conductividad eléctrica. Se calculó el índice de relación de adsorción de sodio (RAS). Se aplicó el método de Pearson entre las variables RAS y la conductividad eléctrica encontrándose una correlación. El RAS fue de 3,94 meq/L y la conductividad eléctrica (CE) de 324 uS/cm. El agua se encuentra en la clasificación C2S1 después de la aplicación del método de Richards; presenta un valor bajo de peligrosidad salina y un valor medio de peligrosidad sódica, considerándose apta para el riego. Los resultados de esta investigación son la base para el desarrollo de un plan de gestión sostenible de los recursos hídricos y manejo adecuado del suelo en la quebrada Togllahuayco. Además, se debe implementar un enfoque que permita la recuperación ecológica de la quebrada evitando la erosión y desgaste del suelo.
\end{abstract}

Palabras clave: Seguridad hídrica, Correlación, Conductividad, Disponibilidad de agua, RAS

\begin{abstract}
Water used for agronomic purposes has significant effects on crop production as well as on the chemical soil deterioration. This study assesses the quantity and quality of water available for irrigation of a sustainable quinoa crop in the Togllahuayco gorge in Guangopolo parish, Ecuador. This place has a volume of $23.347,95 \mathrm{~m}^{3}$ per year of water and only requires $6747,5 \mathrm{~m}^{3}$ per year for quinoa cultivation. Water samples were taken, and the concentration of sodium, potassium, calcium, magnesium, carbonates, bicarbonates, sulfates, chlorides and electrical conductivity were measured. The sodium adsorption ratio index (SAR) was calculated. The Pearson method between RAS variables and electrical conductivity was applied by finding a correlation. The RAS was $3.94 \mathrm{meq} / \mathrm{L}$ and the electrical conductivity (EC) was $324 \mathrm{uS} / \mathrm{cm}$. The water is in the C2S1 classification after application of the Richards method presenting a low value of saline hazard and an average value of sodium hazard, being suitable for irrigation. The results of this research are the basis for the development of a sustainable water resources management plan and adequate soil management in this place. In addition, an approach should be implemented that allows ecological recovery of the territory avoiding soil erosion and wear.
\end{abstract}

Keywords: Water security, Correlation, Conductivity, Water availability, SAR 


\section{Introducción}

Garantizar la disponibilidad de agua representa un objetivo fundamental para todos los países a nivel mundial, ya que el agua es un recurso imprescindible para el desarrollo de la vida (Ortiz \& Sánchez, 2018). En general, las cuencas hidrográficas de montaña presentan graves problemas de deterioro ecológico y erosión de los suelos, debido a la deforestación, prácticas agrícolas inadecuadas en las laderas y exceso de pastoreo, que pueden tener efectos devastadores para los habitantes de las zonas rurales dependientes de la agricultura (FAO, 2007b), además provocan alteraciones en el nivel de fertilidad del suelo y consecuentemente en su capacidad de sostener una agricultura productiva (Prado Wildner y Veiga, 1994). Los volúmenes disponibles de agua dulce para uso agrícola y urbano-industrial a nivel mundial han disminuido considerablemente debido al uso excesivo de aguas superficiales y subterráneas destinadas al riego agrícola para la producción de alimentos de una población en constante crecimiento (Villacrés, 2011). La escasez de agua está vinculada a la seguridad alimentaria, por lo que el sistema de riego desempeña un papel importante en la seguridad alimentaria y el ingreso sostenible, específicamente en los países en desarrollo (Irfan et al., 2014).

\subsection{Diagnóstico inicial}

La calidad del agua en la quebrada Togllahuayco se ve afectada por el deterioro de la vegetación, la deforestación, la erosión del suelo (con una de las tasas más altas del Ecuador), la introducción de especies exóticas, uso de nacientes de agua como bebederos de ganado y disponibilidad estacional del cauce (Peña, 2017). Además, esto implica que las 700 personas que conforman la comunidad ancestral de La Toglla, ubicada en las faldas del cerro Ilaló a $2.630 \mathrm{~m}$ s.n.m., no puedan aprovechar el agua que circula por la quebrada (GAD Parroquial de Guangopolo, 2014; Palacios Orejuela, Ushiña Huera, \& Carrera Villacrés, 2018).

\subsection{Requerimientos de agua para riego}

La cantidad de agua para riego depende de las necesidades hídricas de los cultivos y del agua que está disponible para los mismos de forma natural (Carrera-Villacrés et al., 2018). Sin embargo, en las aguas naturales, de las cuales se toma para riego, pueden presentarse contaminantes de naturaleza inorgánica, especialmente fertilizantes y metales pesados, los cuales tienen importancia por su toxicidad para los organismos vivos; además, el agua superficial a medida que circula por la corteza terrestre arrastra y disuelve minerales, gases, compuestos orgánicos, microorganismos, entre otros (Millán, 2016).

\subsection{Análisis de la química del agua para riego}

En aguas para uso agrícola la calidad es definida por la concentración de iones específicos $\mathrm{Ca}^{2+}, \mathrm{Mg}^{2+}$, $\mathrm{Na}^{+}$y K${ }^{+}$como cationes; $\mathrm{CO}_{3}{ }^{2-}, \mathrm{Cl}^{-}$y $\mathrm{SO}_{4}{ }^{2-}$ como aniones, y otros de menor proporción, como el $\mathrm{B}^{3+}$, I' y $\mathrm{NO}_{3}^{-}$(Amaya Arévalo, 2015; Rey et al., 2007), la conductividad eléctrica (CE) también es útil para determinar la concentración total de sales solubles. La $\mathrm{CE}$ y el $\mathrm{Na}^{+}$son dos parámetros fundamentales que definen la aptitud del agua para riego. El alto contenido de sales en el agua de irrigación genera un aumento de la presión osmótica en la solución del suelo, disminuyendo la adsorción de agua por parte de las plantas. Las sales, además de afectar directamente el crecimiento de las plantas, afectan la constitución del suelo, su permeabilidad y estructura, afectando indirectamente el crecimiento de la planta (Gómez, 2009). El criterio de clasificación de la calidad del agua de riego según Richards es apropiado para zonas áridas y de escaso desarrollo genético; se usó este método debido a que las condiciones climáticas de la zona son similares a las estudiadas con el método propuesto (EMAAP-Q \& Programa de Saneamiento Ambiental, 2006), es decir, corresponde a un área con tendencia a ser una zona árida (FAO, 2007a) y con escasa vegetación. Además, esta metodología se implementó debido a que permite la utilización de un nomograma para determinar la clasificación del agua de riego de acuerdo con los índices CE y RAS (Roblez \& Bautista, 2002), dos de los parámetros más importantes para determinar la cantidad del agua para riego agrícola (Sarabia et al, 2011).

\subsection{Tipo de cultivo a implementar}

La quinua es un grano de alta calidad nutritiva y gran capacidad de adaptabilidad a condiciones adversas de clima y suelo (FAO/RLC, 2011). En la última década la quinua ha ganado un espacio en los mercados de consumo a nivel internacional, lo cual abre oportunidades económicas para los productores andinos del Ecuador (Jacobsen \& Sherwood, 2002). El cultivo de quinua prefiere un suelo franco, con buen drenaje y alto contenido de materia orgánica, con pendientes moderadas y un contenido medio de nutrientes, puesto que la planta es exigente en nitrógeno y calcio, moderadamente en fósforo y poco 
en potasio (Mujica et al., 2001). Puede adaptarse a suelos franco-arenosos, arenosos o franco-arcillosos, siempre que se le dote de nutrientes y no exista la posibilidad de encharcamiento del agua, pues es muy susceptible al exceso de humedad sobre todo en los primeros estados (Gómez \& Aguilar, 2016).

La principal problemática para el pueblo ancestral de La Toglla es la falta de aprovechamiento del recurso hídrico, necesario para la recuperación ecológica de la quebrada Togllahuayco que se ha visto afectada por la erosión del suelo y malas prácticas ambientales, además de que se desconoce la calidad y cantidad de agua disponible para el uso agrícola. El objetivo del presente estudio fue establecer la aptitud y cantidad de agua disponible para riego del cultivo sustentable de quinua, así como la determinación de relaciones entre cationes, aniones y la conductividad eléctrica a través de análisis fisicoquímicos, estadísticos y matemáticos usando el software R.

\section{Materiales y métodos}

\subsection{Determinación de la zona de estudio}

El presente estudio fue de tipo experimental, descriptivo y correlacional; para ello se empleó la NTE INEN 2176:1998. Calidad del agua. Técnicas de muestreo. Se utilizó la técnica de muestreo puntual recomendada para la investigación de contaminantes y calidad del agua. Posteriormente se empleó la NTE INEN 2169:98. Manejo y conservación de muestras. Se eligió el recipiente apropiado, en este caso envase de plástico y para el análisis microbioló- gico recipiente esterilizado. La muestra se conservó en refrigeración a $4^{\circ} \mathrm{C}$. Las muestras fueron tomadas en puntos representativos, es decir, vertientes de agua, coyunturas de flujos hídricos o puntos que presenten características organolépticas desfavorables. Estos puntos se tomaron en las partes alta, media y baja de la quebrada para determinar los cambios en la calidad del agua durante el trayecto desde el nacimiento del agua hasta la desembocadura en el río San Pedro, como se muestra en la Tabla 1.

En la Figura 1 se presenta la zona de estudio de la quebrada Togllahuayco ubicada en la parroquia Guangopolo del cantón Quito, la cual ocupa 551 hectáreas en las faldas del Ilaló; está limitada por las parroquias de Tumbaco, Alangasí y La Merced. El mapa fue elaborado en software ArcGIS Desktop 10.3.1 en el Laboratorio de Cómputo de la Facultad de Ingeniería en Geología, Minas, Petróleos y Ambiental. La altitud media de la quebrada Togllahuayco es de $2.570 \mathrm{~m}$ s.n.m. El clima de la zona de estudio es característico de la zona interandina ecuatorial: mesotérmico húmedo, con una precipitación media anual que varía desde 400 a $800 \mathrm{~mm}$. La temperatura media oscila entre $14.6{ }^{\circ} \mathrm{C}$ a $16.7^{\circ} \mathrm{C}$ (GAD Parroquial de Guangopolo, 2014; INAMHI, 2017). La época caliente se presenta durante los meses de junio a septiembre y se caracteriza por una sequía algo prolongada, fuertes vientos y aumentos de temperatura; en cambio en los meses de mayores lluvias aumenta la humedad siendo estas torrenciales y continuas (GAD Parroquial de Guangopolo, 2014). A continuación, en la Tabla 1 se presenta la ubicación de los puntos de muestreo realizados en la quebrada Togllahuayco.

Tabla 1. Ubicación de los puntos de muestreo

\begin{tabular}{|c|c|c|c|c|}
\hline \multirow[t]{2}{*}{ muestra } & \multirow[t]{2}{*}{ Puntos de Referencia } & \multicolumn{2}{|c|}{$\begin{array}{l}\text { Coordenadas } \\
\text { UTM WGS84 }\end{array}$} & \multirow{2}{*}{$\begin{array}{c}\text { Altura } \\
\text { (msnm) }\end{array}$} \\
\hline & & $\mathrm{X}$ & Y & \\
\hline M1 & Cumbre & 786866 & 9971135 & 2.785 \\
\hline M2 & Parte alta del Ilaló pluvial & 786767 & 9971173,5 & 2.730 \\
\hline M3 & $\begin{array}{l}\text { Mitad del cráter sector } \\
\text { ganadero pluvial }\end{array}$ & 786057 & 9971673 & 2.532 \\
\hline M4 & Parte baja del cráter pluvial & 785426 & 9972205 & 2.430 \\
\hline M5 & Parte baja del cráter pluvial 2 & 785342 & 9972257 & 2.420 \\
\hline M6 & $\begin{array}{l}\text { Estadio de la quebrada de Toglla- } \\
\text { huayco }\end{array}$ & 785070 & 9972445 & 2.400 \\
\hline M7 & Río San Pedro & 784044 & 9972932 & 2.355 \\
\hline M8 & Río San Pedro 2 & 784130 & 9972977 & 2.377 \\
\hline
\end{tabular}




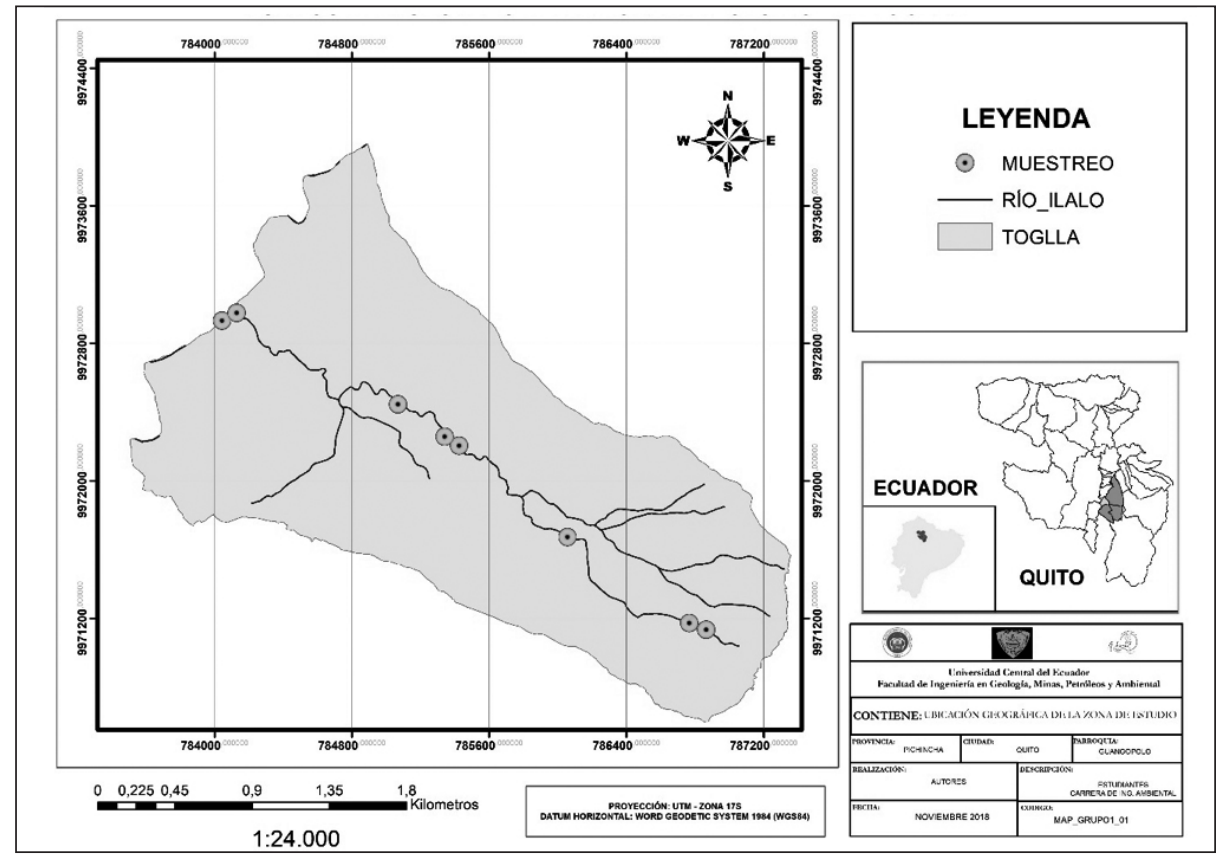

Figura 1. Ubicación de los puntos de muestreo en la quebrada "Togllahuayco" (Fuente: Mapa elaborado por los autores con información obtenida del IGM).

Las muestras se analizaron en el laboratorio químico de la carrera de Ingeniería Ambiental de la Universidad Central del Ecuador. Los parámetros medidos durante el muestreo fueron: cationes de sodio, potasio, calcio, magnesio con el equipo AA espectrofotómetro de absorción atómica PINAACLE 900T y aniones como: carbonatos, bicarbonatos, cloruros con el método de titulación, además, sulfatos, nitratos, fosfatos se midieron con espectrofotómetro HACH DR 4000V, el equipo fue calibrado y certificado por una empresa acreditada. El cálculo de incertidumbre expandida se realizó en base a la guía OAE G02 R00 multiplicando la incertidumbre típica por el factor de cobertura $(\mathrm{k}=2)$ que corresponde a una probabilidad de cobertura de aproximadamente el 95\% conforme al documento EA 4/02. La incertidumbre de calibración del equipo es de 0.0021 Abs
También se midieron variables como el $\mathrm{pH}$ y la temperatura con el potenciómetro marca HANNA modelo HI2210-0 el cual fue calibrado el 15 de enero de 2018. Los sólidos disueltos totales se los midió por gravimetría con la ayuda de la estufa SLN 115 marca POL-EKD APARATURA y con la norma mexicana NOM-AA-341981 (Dirección General de Normativa Mexicana, 1981) y para la determinación de la conductividad eléctrica se midió con el equipo Thermo Scientific Orion Star A212 de acuerdo con los estándares presentados en la investigación de Carrera et al. (2015). En la Tabla 2 se presenta un resumen de los métodos y referencias empleados. En la Tabla 3 se presentan los resultados de las mediciones realizadas en laboratorio de los análisis fisicoquímicos para las muestras de agua

Tabla 2. Métodos y referencias de los análisis fisicoquímicos para las aguas

\begin{tabular}{ccc}
\hline Determinación & Método & Referencia \\
\hline $\begin{array}{c}\mathrm{pH} \\
\begin{array}{c}\text { Conductividad } \\
\text { eléctrica } \\
\text { Temperatura }\end{array}\end{array}$ & $\begin{array}{c}\text { Potenciómetro marca HANNA modelo HI2210-01 } \\
\text { Conductímetro de mesa marca ThermoScientificOrionStar A212 }\end{array}$ & $\begin{array}{c}\text { Standard Methods 4500- } \\
\text { Standard Methods 2510 B }\end{array}$ \\
$\begin{array}{c}\text { Sólidos disueltos } \\
\text { totales }\end{array}$ & $\begin{array}{c}\text { Gravimetría utilizando estufa midió con el equipo estufa SLN 115 } \\
\text { marca POL-EKD APARATURA }\end{array}$ & Standard Methods 2550 B \\
$\begin{array}{c}\text { Cationes - } \\
\text { Cationes - }\end{array}$ & Espectrofotómetro de absorción atómica PINAACLE 900T & NOM-AA-341981 \\
& Espectrofotómetro de absorción atómica PINAACLE 900T & Standard Methods 3500- Na- B
\end{tabular}




\begin{tabular}{|c|c|c|}
\hline Cationes - & Espectrofotómetro de absorción atómica PINAACLE 900T & Standard Methods 3500- Ca- B \\
\hline Cationes - & Espectrofotómetro de absorción atómica PINAACLE 900T & Standard Methods 3500- Mg- B \\
\hline Aniones & Espectrofotómetro HACH DR 4000V & Standard Methods 500-P A, C y E \\
\hline Aniones & Espectrofotómetro HACH DR 4000V & Standard Methods 500-- B \\
\hline Aniones & Espectrofotómetro HACH DR 4000V & Standard Methods 4500- -E \\
\hline Aniones & $\begin{array}{l}\text { Mohr. Titulación con nitrato de plata, Merck, nitrato de plata en } \\
\text { solución } 0.01 \mathrm{~N} \text {. Indicador cromato de potasio al } 5 \%\end{array}$ & Standard Methods 250- Cl E \\
\hline Aniones - & Volumétricos titulación con ácido sulfúrico $0.01 \mathrm{~N}$ & APHA método 2330-B, D \\
\hline Aniones & $\begin{array}{c}\text { Volumétrica titulación con ácido sulfúrico } 0.01 \mathrm{~N} \text {, indicador } \\
\text { fenolftaleína }\end{array}$ & APHA método 2330-B, D \\
\hline
\end{tabular}

Tabla 3. Resultado de los análisis fisicoquímicos de las muestras de agua

\begin{tabular}{|c|c|c|c|c|c|c|c|c|c|}
\hline \multirow{2}{*}{ Parámetros/ Expresados } & \multirow{2}{*}{ Unidad } & \multicolumn{8}{|c|}{ Número de muestra de aguas analizadas, de los parámetros fisicoquímicos } \\
\hline & & M1 & M2 & M3 & M4 & M5 & M6 & M7 & M8 \\
\hline $\mathrm{pH}$ & - & 7,74 & 7,75 & 7,89 & 7,93 & 7,80 & 8,18 & 7,61 & 7,55 \\
\hline Conductividad Eléctrica & $\mathrm{uS} / \mathrm{cm}$ & 161,0 & 187,2 & 232,0 & 307,7 & 306,1 & 270,7 & 543,3 & 583,7 \\
\hline STD & Ppm & 268,0 & 180,0 & 192,0 & 253,2 & 233,3 & 336,0 & 560,0 & 536,0 \\
\hline Temperatura & ${ }^{\circ} \mathrm{C}$ & 15,0 & 15,0 & 14,0 & 15,0 & 16,0 & 18,0 & 19,4 & 19,3 \\
\hline Cationes $-\mathrm{Na}^{+}$ & $\mathrm{meq} / \mathrm{L}$ & 0,670 & 0,920 & 1,170 & 1,290 & 1,400 & 1,140 & 3,498 & 4,391 \\
\hline Cationes - $\mathrm{K}^{+}$ & meq/L & 0,124 & 0,250 & 0,092 & 0,412 & 0,444 & 0,123 & 0,473 & 0,309 \\
\hline Cationes - $\mathrm{Ca}^{2+}$ & $\mathrm{meq} / \mathrm{L}$ & 0,510 & 0,230 & 0,340 & 0,621 & 0,637 & 1,108 & 0,503 & 0,330 \\
\hline Cationes $-\mathrm{Mg}^{2+}$ & $\mathrm{meq} / \mathrm{L}$ & 0,178 & 0,300 & 0,452 & 0,428 & 0,328 & 0,207 & 0,435 & 0,418 \\
\hline Aniones - P-PO ${ }_{4}^{3-}$ & $\mathrm{mg} / \mathrm{L}$ & 0,847 & 0,958 & 0,593 & 0,700 & 0,746 & 0,636 & 7,230 & 2,007 \\
\hline Aniones - $\mathrm{NO}_{3}^{2-}$ & $\mathrm{mg} / \mathrm{L}$ & 0,300 & 3,200 & 0,300 & 0,400 & 0,400 & 0,400 & 5,500 & 4,100 \\
\hline Aniones - $\mathrm{SO}_{4}^{2-}$ & $\mathrm{meq} / \mathrm{L}$ & 0,000 & 0,006 & 0,029 & 0,744 & 0,756 & 0,685 & 0,377 & 0,800 \\
\hline Aniones - $\mathrm{Cl}^{-}$ & $\mathrm{meq} / \mathrm{L}$ & 0,680 & 1,200 & 0,950 & 0,800 & 0,400 & 0,400 & 0,300 & 1,450 \\
\hline Aniones - $\mathrm{HCO}_{3}^{2-}$ & $\mathrm{meq} / \mathrm{L}$ & 0,800 & 0,500 & 1,080 & 1,200 & 1,650 & 1,490 & 4,300 & 3,154 \\
\hline Aniones - $\mathrm{CO}_{3}^{2-}$ & $\mathrm{meq} / \mathrm{L}$ & 0,000 & 0,000 & 0,000 & 0,000 & 0,000 & 0,000 & 0,000 & 0,000 \\
\hline$\sum$ cationes & $\mathrm{meq} / \mathrm{L}$ & 1,482 & 1,700 & 2,054 & 2,751 & 2,809 & 2,577 & 4,909 & 5,449 \\
\hline$\sum$ aniones & meq/L & 1,480 & 1,706 & 2,059 & 2,744 & 2,806 & 2,575 & 4,977 & 5,404 \\
\hline Error & $\%$ & 0,076 & 0,183 & 0,127 & 0,132 & 0,052 & 0,024 & 0,688 & 0,413 \\
\hline
\end{tabular}

\subsection{Descripción de la metodología}

Para analizar el problema con respecto a la cantidad de agua disponible para el cultivo y la aptitud del agua para riego se realizó una relación lineal con el método de correlación de Pearson mediante el uso del software estadístico R Studio, entre las variables del índice de relación de adsorción de sodio (RAS), los cationes y aniones en contraste con la conductividad eléctrica $(\mathrm{CE})$.

\subsection{Relación de adsorción de sodio (RAS)}

Para determinar la calidad de agua que se encuentra en la quebrada Togllahuayco, con los datos de concentraciones de cationes de $\mathrm{Ca}^{2+}, \mathrm{Mg}^{2+}$ y $\mathrm{Na}^{+}$ en meq/L, es posible determinar la sodicidad, relación de adsorción de RAS (Puñales \& Aguilar, 2016) a partir de la ecuación [1]:

$$
R A S=\frac{N a^{+}}{\sqrt{\left(C a^{2+}+M g^{2+}\right) / 2}}
$$

Se utilizó el software estadístico "R Studio" para calcular indicadores como: coeficientes de correlación, error estándar y R cuadrado; y analizar la relación que existe entre los factores RAS, altitud, cationes, aniones y CE, mediante la aplicación de técnicas como correlación, modelos de regresión lineal para facilitar la comprensión entre variables 
(Carrera-Villacrés et al., 2018), el cálculo de $\mathrm{R}^{2}$ se lo realizó a partir de la ecuación [2]

$$
R^{2}=\frac{\sum_{t=1}^{T}\left(\hat{Y}_{t}-\bar{Y}\right)^{2}}{\sum_{t=1}^{T}\left(Y_{t}-\bar{Y}\right)^{2}}
$$

Donde:

$$
\begin{aligned}
& \hat{\mathrm{Y}}_{\mathrm{t}}=\text { varianza } \\
& \overline{\mathrm{Y}}=\text { media } \\
& \mathrm{Y}_{\mathrm{t}}=\text { valor de } \mathrm{y}
\end{aligned}
$$

\subsection{Diagrama de Richards}

El diagrama de Richards para la clasificación de aguas de riego sirve para determinar el nivel de peligrosidad por sodio y por la salinidad presente en el agua y como datos de entrada se coloca el (RAS) y la conductividad eléctrica de las muestras analizadas.

Al hacer uso de los criterios tradicionales de la relación de adsorción de sodio y la conductividad eléctrica, se clasificaron las muestras como C1, C2 y C3, según las directrices del laboratorio de salinidad de EE. UU. (Can et al., 2008). La clasificación C1 son suelos y aguas satisfactorias para riego con respecto a la concentración de sales; sin embargo, las aguas $\mathrm{C} 2$ podrían impactar de manera adversa a cultivos sensibles, en el caso de los $\mathrm{C} 3$ se puede obtener un rendimiento adecuado de los cultivos, siempre y cuando se haga un manejo adecuado de los suelos y el drenaje sea eficiente, de lo contrario se podrían presentar condiciones de alta salinidad.

La peligrosidad por salinidad se representa con la letra S y acorde al nivel de peligrosidad se acompaña del número; para un nivel de peligro bajo se tiene la categoría $\mathrm{S} 1$ y para el nivel de categoría muy alto se tiene S4 (Can et al., 2008).

\subsection{Cálculo de disponibilidad de agua y la deman- da de agua para cultivo de quinua}

Para la determinación de la disponibilidad del agua se utilizó el balance hídrico a partir de variables como la temperatura y precipitación otorgado por el INAMHI de la estación "La TolaM002", siendo la estación meteorológica más cercana a la zona de estudio para el periodo 1990-2017 cuyos datos fueron procesados gracias al software Excel y por el método de Thorthwaite (Ordóñez, 2011).

a) Disponibilidad de agua. Se ve reflejada directamente de la precipitación, pero no toda el agua es utilizable debido a que un porcentaje se escurre a través de la quebrada, otro porcentaje se infiltra o se evapora. Es importante conocer la cantidad de agua que es útil para las plantas conocida como precipitación efectiva y es aquella que logra satisfacer al menos una parte de los requerimientos hídricos (Alfaro, 2012).

Para el cálculo de la precipitación efectiva se utilizó el método recomendado por la Organización de las Naciones Unidas para la alimentación (FAO) y se denomina Método del Servicio de Conservación de Suelos del Departamento de Agricultura de los Estados Unidos (USDA S.C.), que se especifica en la ecuación [3] (Nieto et al., 2018).

$$
\begin{aligned}
& \text { si } \mathrm{P}<250 \mathrm{~mm} \\
& \text { si } \mathrm{P}>250 \mathrm{~mm} \\
& P e=\frac{P}{125} *(125-0.2 P) \text { si } \mathrm{P}<250 \mathrm{~mm} \\
& P e=(125+0.1 P) \text { si } \mathrm{P}>250 \mathrm{~mm}
\end{aligned}
$$

En la Tabla 4 se muestra los resultados de la precipitación media mensual y la precipitación efectiva utilizando la fórmula cuando $\mathrm{P}<250 \mathrm{~mm}$ en todos los meses.

Tabla 4. Precipitación efectiva en la quebrada Togllahuayco

\begin{tabular}{ccc}
\hline Mes & Precipitación $(\mathrm{mm})$ & $\begin{array}{c}\text { Precipitación } \\
\text { efectiva }(\mathrm{mm})\end{array}$ \\
\hline Enero & 69.9 & 62.1 \\
Febrero & 77.3 & 67.7 \\
Marzo & 123.2 & 98.9 \\
Abril & 114.5 & 93.5 \\
Mayo & 70.2 & 62.3 \\
Junio & 27.5 & 26.3 \\
Julio & 13.9 & 13.6 \\
Agosto & 16.4 & 16.0 \\
Septiembre & 47.1 & 43.5 \\
Octubre & 104.7 & 87.2 \\
Noviembre & 102.9 & 85.9 \\
Diciembre & 78.3 & 68.5 \\
Total & 845.9 & 725.6 \\
\hline
\end{tabular}


b) Requerimiento hídrico de cultivos. La evapotranspiración se la realizó a través del método de Thorthwaite (Ordóñez, 2011), utilizando la temperatura media mensual obtenida de los datos meteorológicos proporcionados por el INAMHI y las constantes de Aparicio juntamente con el software Hidroesta para su procesamiento.

La evapotranspiración por cultivo (ETc) para la quinua se la calculó a través del coeficiente de cultivo, acorde con la investigación reportada por García, Raes, \& Jacobsen (2001) los coeficientes para la quinua son para la fase inicial 0,52, para la fase de maduración $1 \mathrm{y}$ para la fase de cosecha 0,70 y relacionando con la evapotranspiración potencial se tiene la ecuación [4]

$$
E T c=k c * E T o
$$

Donde:

$\mathrm{ETc}=$ Evapotranspiración del cultivo en $\mathrm{mm} / \mathrm{mes}$

$\mathrm{Kc}=$ coeficiente del cultivo

$\mathrm{ETo}=$ Evapotranspiración potencial $\mathrm{mm} / \mathrm{mes}$

Para determinar la eficiencia del aprovechamiento de agua para riego se realiza el balance de la entrada de agua disponible y el requerimiento hídrico del sistema (Nieto et al., 2018) tomando en cuenta el área de superficie a ser regada. Acorde con la FAO para el cultivo de quinua es recomendable la siembra una vez al año con un periodo de 6 meses empezando de enero a julio (Gòmez \& Aguilar, 2016).

\section{Resultados y discusión}

\subsection{Disponibilidad de agua y requerimiento hidri- co para el cultivo de quinua}

Para determinar la disponibilidad del agua es indispensable conocer el área de la quebrada que va a recolectar el agua de las precipitaciones. Esta se calculó gracias a sistemas de información geográfica obteniéndose un área de $5.5^{*} 10^{5} \mathrm{~m}^{2}$ y acorde a la entrevista con el representante de la comunidad ancestral "La Toglla" quien planea implementar el proyecto (Dehousse, 2018) manifestó que el área destinada para la agricultura sería de $25.000 \mathrm{~m}^{2}$. A continuación, en la Tabla 5 se presenta el cuadro resumen de la demanda y en la Tabla 6 se presenta la oferta de agua para riego así como el balance hídrico en la Tabla 7.

Tabla 5. Demanda hídrica para cultivo de quinua

\begin{tabular}{lcccc}
\hline \multicolumn{5}{c}{ Demanda hídrica } \\
\hline Mes & Kc & Eto & $\mathbf{E t c / \mathbf { m } 2}$ & $\begin{array}{c}\text { ETc } \\
\text { (Agua requerida) }\end{array}$ \\
& & $\mathbf{m m}$ & $\mathbf{m m} \mathbf{m}^{\mathbf{2}}$ & $\mathbf{m}^{\mathbf{3}}$ \\
Enero & 0,5 & 55,9 & 27,95 & 698,7 \\
Febrero & 0,5 & 50,5 & 25,24 & 631,1 \\
Marzo & 0,5 & 56,5 & 28,23 & 705,7 \\
Abril & 1 & 54,6 & 54,6 & 1366,0 \\
Mayo & 1 & 56,5 & 56,5 & 1411,5 \\
Junio & 1 & 54,6 & 38,24 & 956,2 \\
Julio & 0,7 & 55,9 & 39,13 & 978,3 \\
Total & & & & 6747.5 \\
\hline
\end{tabular}

Tabla 6. Oferta hídrica

\begin{tabular}{ccc}
\hline \multicolumn{3}{c}{ Oferta hídrica } \\
\hline Mes & $\begin{array}{c}\text { Precipitación efectiva } \\
\mathbf{m m} / \mathbf{m}^{\mathbf{2}}\end{array}$ & $\begin{array}{c}\text { Agua disponible } \\
\mathbf{m}^{3}\end{array}$ \\
Enero & 62,10 & 3415,60 \\
Febrero & 67,74 & 3725,82 \\
Marzo & 98,93 & 5441,26 \\
Abril & 93,53 & 5144,54 \\
Mayo & 62,32 & 3428,09 \\
Junio & 26,31 & 1447,02 \\
Julio & 13,55 & 745,62 \\
\hline
\end{tabular}

Tabla 7. Balance general del recurso hídrico

\begin{tabular}{cccc}
\hline \multicolumn{4}{c}{ Balance hídrico } \\
\hline Mes & $\begin{array}{c}\text { Agua } \\
\text { disponible } \\
\text { (oferta) }\end{array}$ & $\begin{array}{c}\text { Requerimiento } \\
\text { de agua } \\
\text { (demanda) }\end{array}$ & Balance \\
Enero & 3415,6 & $\mathbf{m}^{3}$ & $\mathbf{m}^{3}$ \\
Febrero & 3725,8 & 698,7 & 2716,8 \\
Marzo & 5441,3 & 631,1 & 3094,7 \\
Abril & 5144,5 & 705,7 & 4735,5 \\
Mayo & 3428,1 & 1366,0 & 3778,5 \\
Junio & 1447,0 & 956,2 & 2016,5 \\
Julio & 745,6 & 978,3 & $-232,7$ \\
\hline
\end{tabular}




\subsection{Aptitud del agua para riego}

Para la determinación de la aptitud del agua para riego se obtuvo el índice de adsorción de sodio
(RAS) así como la conductividad eléctrica, después de analizar los 8 puntos de muestreo, en la Tabla 8 se evidencia los valores de la conductividad eléctrica y el RAS.

Tabla 8. Conductividad eléctrica y RAS de los puntos de muestreo de la quebrada Togllahuayco

\begin{tabular}{ccc}
\hline $\mathrm{N}^{\circ}$ muestra & $\begin{array}{c}\text { RAS } \\
\mathbf{m e q} / \mathbf{L}\end{array}$ & $\begin{array}{c}\mathrm{CE} \\
\mathbf{u S} / \mathbf{c m}\end{array}$ \\
\hline Punto 1 & 1,62 & 161,0 \\
Punto 2 & 2,53 & 187,2 \\
Punto 3 & 2,63 & 232,0 \\
Punto 4 & 2,52 & 307,7 \\
Punto 5 & 2,85 & 306,1 \\
Punto 6 & 1,99 & 270,7 \\
Punto 7 & 7,22 & 543,3 \\
Punto 8 & 10,15 & 583,7 \\
\hline
\end{tabular}

Un sistema basado en la medida de la conductividad eléctrica del agua para determinar el riesgo de salinización del suelo y en el cálculo de la relación de adsorción de sodio (RAS) para determinar el riesgo de sodificación o alcalinización, es el propuesto por Richards, uno de los sistemas más utilizados en España (Olías, Cerón, \& Fernández, 2005). En Ecuador también se utiliza la clasificación de Richards tal como se evidencia en los estudios realizados por Pérez (2019) y Mencías (2015). Según el diagrama de Richards para la clasificación del agua de riego presentado por el departamento de Agricultura de los Estados Unidos, la figura 2 muestra que el agua de la cuenca hidrográfica de La Toglla se ubica en los campos C1S1, C1S2 Y C2S2.

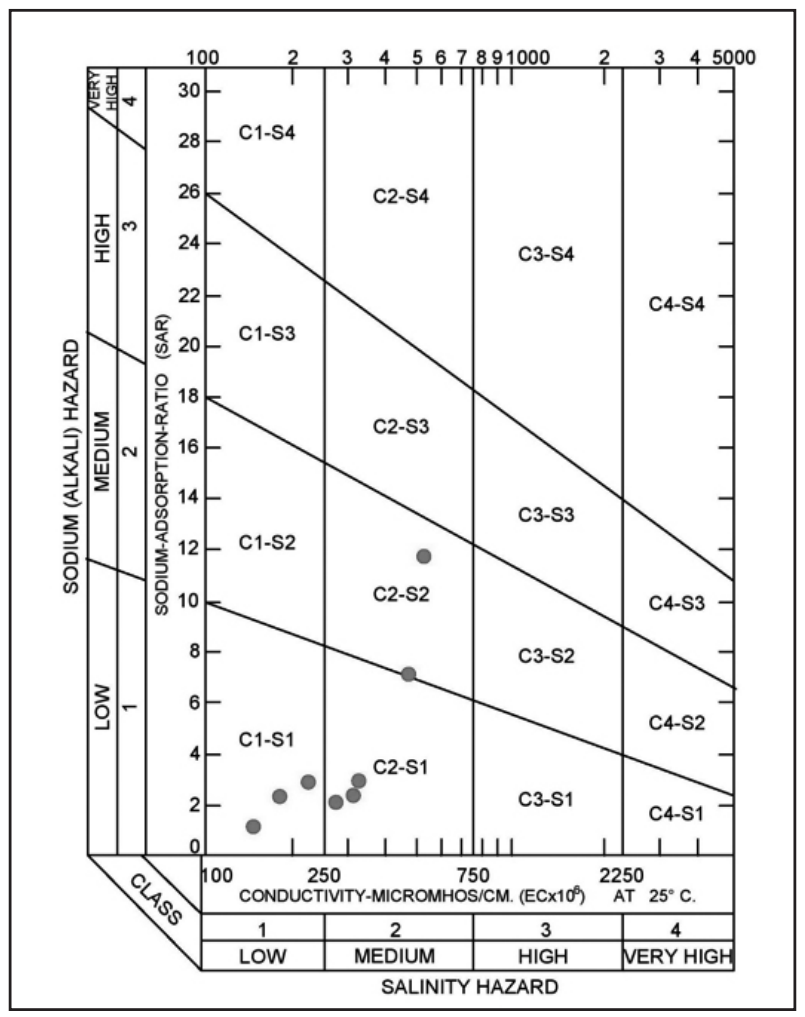

Figura 2. Diagrama Richards para la clasificación del agua para riego (United States Department of Agriculture). 


\subsection{Correlación entre variables}

Se realizó la correlación de variables entre la conductividad eléctrica y la altitud, RAS, cationes y aniones como se muestra en la Tabla 8, para determinar las variables que más se ajusten y expliquen el fenómeno de salinidad y el incremento o decremento de la conductividad eléctrica siendo los parámetros más relevantes para la determinación de la aptitud del agua para riego.

Tabla 8. Resultados de progresión obtenidos en el software R

\begin{tabular}{ccccc}
\hline $\begin{array}{c}\text { Indicadores } \\
\text { estadísticos }\end{array}$ & $\begin{array}{c}\text { Altitud } \\
\text { vs } \\
\text { CE }\end{array}$ & $\begin{array}{c}\text { RAS } \\
\text { vs } \\
\text { CE }\end{array}$ & $\begin{array}{c}\text { Cationes } \\
\text { vs } \\
\text { CE }\end{array}$ & $\begin{array}{c}\text { Aniones } \\
\text { vs CE }\end{array}$ \\
\hline $\begin{array}{c}\text { Coeficiente de } \\
\text { correlación } \\
\mathrm{R}^{2}\end{array}$ & $\mathbf{0 , 5 6 2 1}$ & $\mathbf{0 , 8 8 9 7}$ & $\mathbf{0 , 9 9 7 8}$ & $\mathbf{0 , 9 9 8 6}$ \\
$\begin{array}{c}\text { Estándar } \\
\text { Error }\end{array}$ & 0,2852 & 0,0026 & 0,0001 & 0,0001 \\
$\begin{array}{c}\text { Residual } \\
\text { Standard } \\
\text { Error }\end{array}$ & 118,5 & 1,097 & 0,0742 & 0,0587 \\
\hline
\end{tabular}

En base a los resultados no se presenta una correlación significante entre la altitud y la conductividad eléctrica, al contrario, se presenta una correlación significativamente alta entre el RAS, cationes y aniones con la conductividad eléctrica (CE).

La Figura 3 presenta la gráfica entre el RAS y la CE mostrando una relación directamente proporcional con un $\mathrm{R}^{2}=0.8897$, contrario a la Figura 4 que presenta una relación inversamente proporcional entre la altura y la conductividad eléctrica, aunque presenta un valor insignificante de relación con un $\mathrm{R}^{2}=0.5621$. La Figura 5 presenta la gráfica entre los aniones vs. la conductividad eléctrica con un $\mathrm{R}^{2}=0.9986$ y la Figura 6 presenta la gráfica entre cationes y conductividad eléctrica con un valor significativamente alto con un coeficiente de correlación $\mathrm{R}^{2}=0.9978$

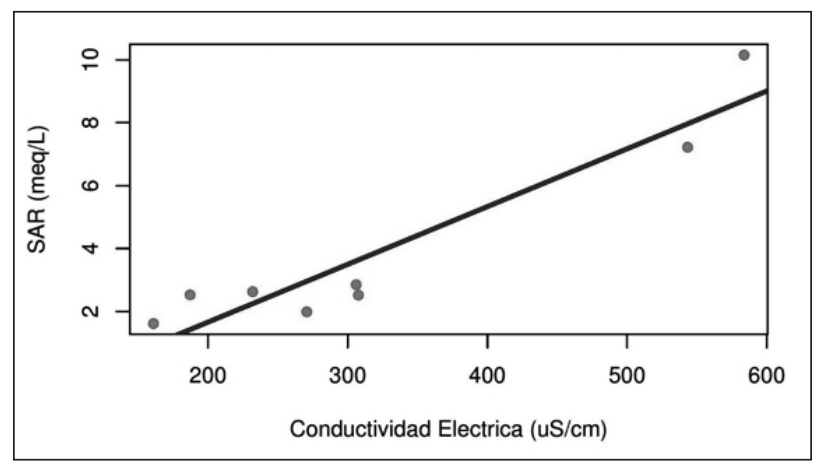

Figura 3. Relación entre RAS y CE.

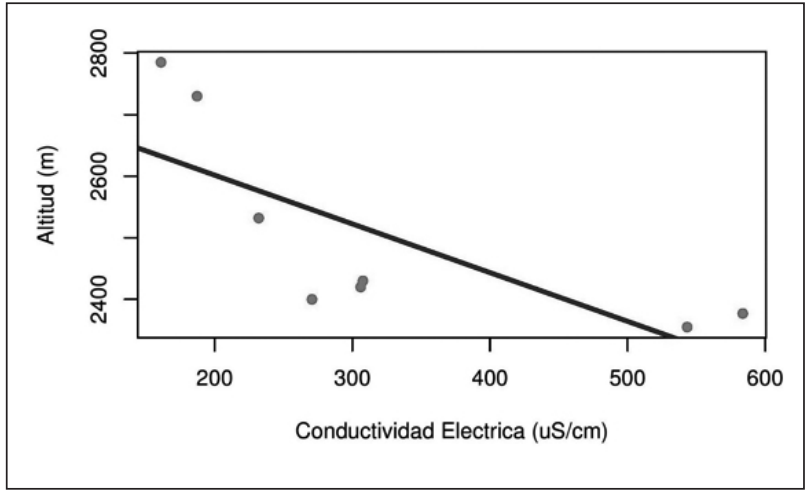

Figura 4. Relación entre altitud y CE.

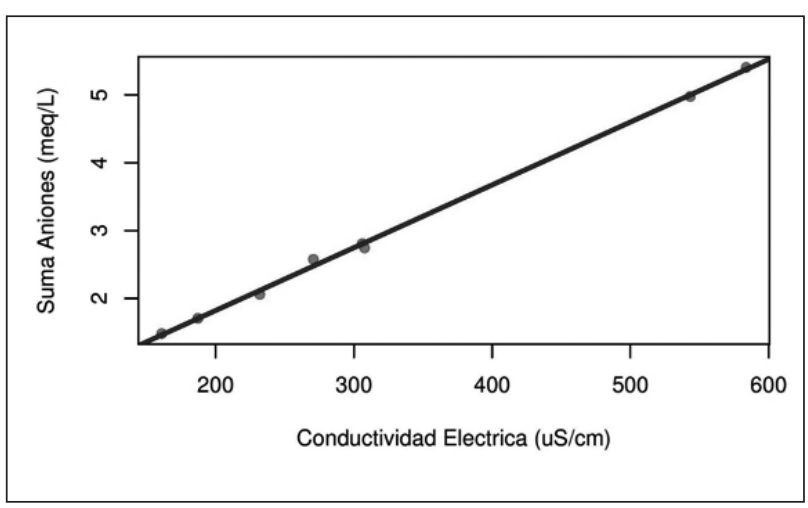

Figura 5. Relación entre RAS y CE.

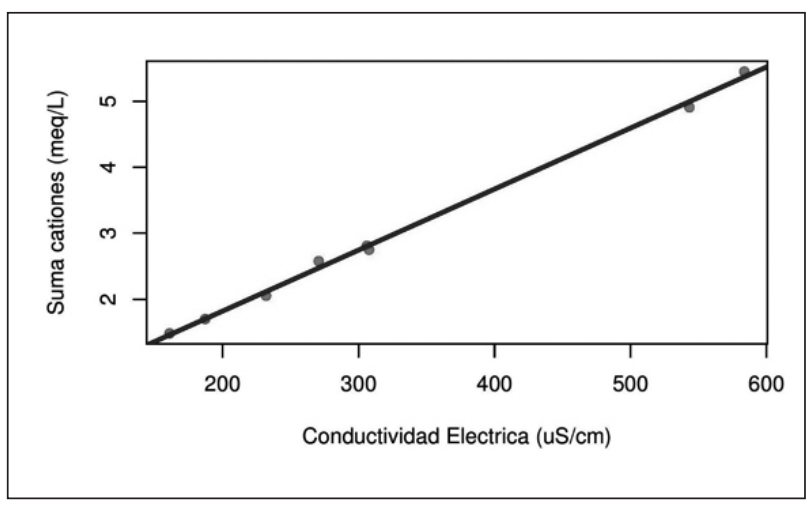

Figura 6. Relación entre RAS y CE.

\section{Discusión}

El recurso hídrico de la quebrada puede ser usado para el riego de cultivos, debido a que los valores de RAS promedio y conductividad eléctrica promedio fueron de $3,94 \mathrm{meq} / \mathrm{L}$ y $324 \mathrm{uS} / \mathrm{cm}$, respectivamente, ubicándose en la clasificación C2S1 el cual indica que el agua presenta un valor bajo de peligrosidad salina y un valor medio de peligrosidad sódica. Los valores de CE son más bajos en 
la parte alta de la cuenca, esto quiere decir que la cantidad de sales es menor y la calidad del agua es excelente, esto se debe a que en la zona alta recién comienza la vertiente natural, mientras el río sigue su cauce los valores de CE van aumentando debido a que entra en contacto con la superficie y se produce un arrastre de cationes y aniones que enriquecen el agua. La CE presenta una mayor influencia tanto en aniones como en cationes, obteniéndose coeficientes de correlación entre estos de 0,9986 y 0,9978 , respectivamente, indicando que a medida que aumenta la CE la concentración de aniones y cationes es mayor, aumentando el riesgo de peligrosidad del agua para riego.

\section{Conclusiones}

La aptitud del agua para riego de la quebrada Togllahuayco se encuentra en la clasificación C2S1, lo que quiere decir que presenta baja peligrosidad salina y media peligrosidad sódica, lo que vuelve muy factible que se utilice el agua para cultivos sin tener repercusiones graves a corto o largo plazo.

El balance hídrico efectuado entre la cantidad de precipitación (oferta) y el requerimiento de agua para el cultivo de quinua (demanda) para el

\section{Referencias}

Alfaro, G. (2012). Diseño de zonas de riego, requerimiento de riego de los cultivos. Universidad Autónoma Agraria Antonio Narro.

Amaya Arévalo, Á. J. (2015). Evaluación hidrogeoquímica e hidroquímica de la subcuenca del río Aranchacal. Universidad de El Salvador.

APHA, AWWA, \& WEF. (1995). APHA Methods: Standard Methods for the Examination of Water and Wasterwater. Washington, DC.

Can, Á.C., Ayala, C.R., Escobar, M.O., López, C.T., \& Díaz, J.C. 2008. Evaluación de la relación de adsorción de sodio en las aguas del río Tulancingo, estado de Hidalgo, México. Terra Latinoamericana. 26(3): 243-252.

Carrera-Villacrés, D., Guerrón Varela, E., Cajas Morales, L., González Farinango, T., Guamán Pineda, periodo de estudio que comprende los meses de enero a julio señala que existe la suficiente cantidad de agua disponible para riego, a excepción del mes de julio que presenta una deficiencia de agua por lo que se recomienda la implementación de un reservorio para almacenar agua para el mes de estiaje o cambiar el periodo de cultivo evitando el mes de sequía.

Existe una relación directamente proporcional entre la conductividad eléctrica (CE) y las variables de relación de absorción de sodio (RAS), cationes y aniones con un valor de coeficiente de relación $\mathrm{R}^{2}$ de $0,89,0,99$ y 0,99 respectivamente lo que quiere decir que a mayor conductividad eléctrica mayor RAS, cationes y aniones lo que conlleva a un incremento de la peligrosidad del agua para riego tanto por salinidad como por sodicidad. La conductividad eléctrica es una variable fácilmente medible en laboratorio o con equipos de campo, y con el resultado de esta investigación, al medir esta variable, se puede deducir el comportamiento de la aptitud del agua para riego y la peligrosidad salina y sódica; en el caso de que se tenga valores muy altos de CE se recomienda realizar un muestreo y los análisis de cationes y aniones para determinar la fuente de ese incremento y poder solucionar de manera técnica.

É., \& Velarde Salazar, P. A. (2018). Relación de temperatura, $\mathrm{pH}$ y $\mathrm{CE}$ en la variación de concentración de fosfatos en el río Grande, cantón Chone. Congreso de Ciencia y Tecnología ESPE, 13(1). https://doi.org/10.24133/cctespe.v13i1.807

Carrera, D., Crisanto, T., Guevara, P., \& Maya, M. (2015). Relación entre la composición química inorgánica del agua, la precipitación y la evaporación en la cuenca de río Grande, Chone, Ecuador. Enfoque UTE, 6(1), 25-34. https://doi. org/10.29019/enfoqueute.v6n1.52

Carrera-Villacrés, D., Ayala, J., Carmona, C., Garófalo, M., Mastián, F., Moyón, Á., Haro, M. (2018). Caracterización hídrica de la cuenca hidrográfica Toglla con fines de conservación de bosques y producción agrícola. En: $M e$ morias del Congreso REDU VI 2018. Ibarra: Editorial Universidad Técnica del Norte. 
Dehousse, P. (2018). Entrevista a la comuna ancestral La Toglla. Quito.

Dirección General de Normativa Mexicana. Normativa Oficial Mexicana NOM-AA-34-1981. (1981). México.

EMAAP-Q, \& Programa de Saneamiento Ambiental (2006). Plan de Manejo del cerro Ilaló. Quito: EMAAP-Q.

FAO. (2007a). Secuestro de carbono en tierras áridas. Roma: FAO, Informes sobre recursos mundiales de suelos 102 .

FAO. (2007b). La nueva generación de programas y proyectos de gestión de cuencas hidrográficas. Roma: Estudio FAO: Montes 150.

FAO/RLC. (2011). La quinua: cultivo milenario para contribuir a la seguridad alimentaria mundial. Santiago: Oficina Regional de la FAO para América Latina y el Caribe (FAO/RLC).

GAD Parroquial de Guangopolo. (2014). Plan de Desarrollo Parroquial 2011-2025. Quito-Ecuador.

García, M., Raes, D., \& Jacobsen, E. (2001). Análisis de la evapotranspiración de referencia en el altiplano boliviano y determinación de los requerimientos de riego de quinua (Chenopodium quinoa Willd). En International Congress on quinoa. Lima, Perú.

Gómez, L., \& Aguilar, E. (2016). Guía de cultivo de la quinua. Lima: FAO, Universidad Nacional Agraria La Molina.

Gómez, M. L. (2009). Definición de la aptitud de agua para riego en un agroecosistema del sur de la provincia de Córdoba, Argentina. Investigación y Ciencia de la Universidad Autónoma de Aguascalientes, 45, 38-43.

Instituto Nacional de Meteorología y Climatología INAMHI (2017). Boletines climáticos y agrícolas. Obtenido de: http://www.serviciometeorologico.gob.ec/

Irfan, M., Arshad, M., Shakoor, A., \& Anjum, L. (2014). Impact of irrigation management practices and water quality on maize production and water use efficiency. Journal of Animal and Plant Sciences, 24(5), 1518-1524.
Jacobsen, S.E., \& Sherwood, S. (2002). Cultivo de granos andinos en Ecuador: Informe sobre los rubros quinua, chocho y amaranto. Quito: Abya-Yala.

Mencías, D. (2015). Determinación de la infiltración en el suelo, estudio de la calidad del agua para regadio y manejo ambiental del sistema de riego El Pisque - Pichincha. Tesis de maestría. Universidad Internacional SEK, Quito, Ecuador.

Millán, F. (2016). Curso de Química II. Unidad 6: Calidad de aguas potabilizables. Instituto Universitario Politécnico Santiago Mariño, Extensión Mérida. https://doi.org/10.13140/ RG.2.1.4267.8000

Mujica, A., Y., Jacobsen, S., Izquierdo, J., \& Marathee, J.P. (2001). Quinua (Chenopodium quinoa willd.) ancestral cultivo andino, alimento del presente y futuro. Santiago: FAO.

Nieto, C., Pazmiño, E., Rosero, S., \& Quishpe, B. (2018). Estudio del aprovechamiento de agua de riego disponible por unidad de producción agropecuaria, con base en el requerimiento hídrico de cultivos y el área regada, en dos localidades de la Sierra ecuatoriana. Siembra, 5(1), 51-70.

Norma Técnica Ecuatoriana INEN (NTE) 2169. (1998). Agua. Calidad del agua. Muestreo. Manejo y conservación de muestras. Quito-Ecuador.

Norma Técnica Ecuatoriana INEN (NTE) 2176. (1998). Agua. Calidad del agua. Muestreo. Técnicas de muestreo. Quito-Ecuador.

Olías, M., Cerón, J. C., \& Fernández, I. (2005). Sobre la utilización de la clasificación de las aguas de riego del U.S. Laboratory Salinity (USLS). Geogaceta, 37, 111-113.

Ordóñez, J. (2011). Cartilla Técnica: Balance hídrico superficial. Lima: Global Water Partnership South America, Sociedad Geográfica de Lima.

Ortiz, D. W., \& Sánchez, J. R. (2018). Caracterización geomorfológica y biofísica de las cuencas de aporte de las captaciones de los sistemas de agua potable de los cantones que conforman la Mancomunidad Cañari. Universidad de Cuenca, Ecuador. 
Palacios Orejuela, I. F., Ushiña Huera, D. P., \& Carrera Villacrés, D. V. (2018). Identificación de cangahuas para su recuperación mediante estudio multicriterio y constatación in situ en comunas del volcán Ilaló. Congreso de Ciencia y Tecnología ESPE, 13(1), 10-13. https://doi. org/10.24133/cctespe.v13i1.649

Peña, J. (2017). Modelo de gestión de las tierras comunitarias para el pueblo ancestral La Toglla-Guangopolo con criterios de sostenibilidad. Universidad de las Fuerzas Armadas (ESPE).

Pérez, A. (2019). Riesgo de sodicidad en los suelos del cantón Milagro, Guayas-Ecuador en época de estiaje. Revista Politécnica, 42(2). https:// doi.org/10.33333/rp.vol42n2.899

Prado Wildner, L. do; Veiga, M. da. (1994). Tema 2: Erosión y pérdida de fertilidad del suelo. En: Erosion de suelos en America Latina. Taller sobre la Utilización de un Sistema de Información Geográfica (SIG) en la Evaluación de la Erosión Actual de Suelos y la Predicción del Riesgo de Erosión Potencial. Santiago: FAO.

Puñales, T.T., \& Aguilar, C.C.B. (2016). La calidad del agua para el riego. Principales indicadores de medida y procesos que la impactan. Agroecosistemas, 4(1), 47-61.

Rey, M., Stincer, R. J., González, F., \& Sarmiento, O. (2007). Results of the application of drips irrigation in the rotation the banana CENSA 3/4 with papaya red $\mathrm{M}$ aradol with high density plantation. Revista Ciencias Técnicas Agropecuarias, 16(3), 9-14.

Roblez, C., \& Bautista, G. (2002). Calidad del agua de riego. Temas de ciencia y tecnología, 6(18), 39-45.

Sarabia, I., Cisneros, R., Aceves, J., Durán, H., \& Castro, J. (2011). Calidad del agua de riego en suelos agrícolas y cultivos del Valle de San Luis Potosí, México. Revista Internacional de Contaminación Ambiental, 27(2), 103-113.

Villacrés, D. C. (2011). Salinidad en suelos y aguas superficiales y subterráneas de la cuenca evaporitica de Río Verde-Matehuala, San Luis Potosí. Montecillo, México. Tesis Doctoral. Colegio de Postgraduados, México. https://doi.org/10.13140/ RG.2.2.14037.73443 